\title{
A Walk-through System for Building Acoustics Evaluation Based on Virtual Environment Technology
}

\author{
Ying Zhang and Terrence Fernando \\ Center for Virtual Environments \\ University of Salford \\ Greater Manchester, M5 4WT, UK \\ Tel: +44 (0) 161 2952906, Tel: +44 (0) 1612952904 \\ Fax: +44 (0) 1612952925 \\ Email: Y.Zhang1@pgr.salford.ac.uk, T.Fernando@salford.ac.uk
}

\begin{abstract}
Virtual Reality technology, especially visualization and auralization, provides a useful method to interactively implement the subjective analysis and evaluation of acoustic properties prior to the actual construction of a building. This paper presents a Virtual Environmentbased walk-through system for interactive acoustic evaluation of buildings within a CAVE environment. It describes the architecture of the system, the system components, schedule algorithm, synchronous integration of acoustic simulation and visualization. The trade-off algorithm of the real time simplified acoustic space simulation by B-format soundfield representation, non real time detailed acoustic space simulation and the comparison between them are also mentioned. Some cases such as airport, cinema, boardroom, underground station and classroom are studied in the two simulation methods respectively. The potential applications of this system are presented as well.
\end{abstract}

Keywords: Virtual Environment, Walk-through System, Visualization, Auralization, Interactivity, Acoustic Simulation.

\section{Introduction}

The subjective analysis and evaluation of acoustic properties prior to the actual construction of a building is a very important aspect during the design stage. There have been many occasions where construction teams have had to modify buildings in order to achieve the expected acoustic properties, resulting in extra high cost and delay in completing the construction. Therefore, a good evaluation system is necessary to support this process during buildings design for acoustic consideration. However, software tools currently available for architectural acoustic design and simulation use 2D user interfaces to communicate acoustic properties to the users. Input files are used to describe parameters such as the size, surface materials, sources positions/properties and receivers positions/directions etc. The prediction outputs are text format files, graphs, charts, and tables. These have some limitations. For example, the client and consumer of the construction projects cannot understand the graphs, abstract data, charts and tables well, or even visualized output. Virtual Reality (VR) technology, especially visualization and auralization, provides a useful method to interactively implement this task. This paper presents a Virtual Environment-based walk-through system for interactive acoustic evaluation of buildings within a Cave Automatic Virtual Environment (CAVE).

With visualization, binaural room acoustic simulation techniques and real time auralization components, this system provides an immersive Virtual Environment (VE) in which the clients can be immersed within the proposed design and interact with the space to make design changes and experience the effect of that modification, especially acoustic properties. The working process of the system is shown in Fig. 1. New 3D CAD building geometric design and its acoustic requirements are imported into the system. This system uses the geometric data and the relevant acoustic model generated to implement visualization and auralization via VR facilities. The user can be immersed within the virtual environment to hear and see the aural/visual effect of the spaces. If it does not reach the requirements, the user can modify the parameters like surface materials, space layout, or sound source characteristics and repeat above steps till satisfied with its final design or layout. As for cases of a concert, boardroom, auditorium, cinema and studio in this system, the user is able to place himself or herself at different positions to feel the sound effect for critical evaluation by non-real time simulation algorithm. For the airport, underground station, factory shop, the user can experience real time walking from room to room (walk-through) and its sound effect via real time simplified acoustic space simulation that uses the trade-off algorithm for B-format soundfield representation. In this way, interactive simulation and evaluation can be implemented.

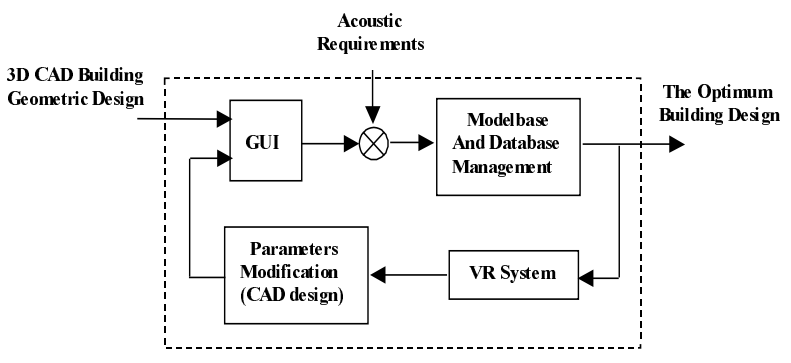

Fig. 1 The Process of Decision-making 
The motivation for the research emerged from the rapidly growing applications and interests in the virtual reality [14], increasing trend to use virtual reality technology in decision making system [13], a variety of approaches for modeling acoustic performance $[2][3][4][5]$ and available techniques not support real time interactive acoustic design and simulation $[6][7][11][12]$, also challenges inherent in achieving desirable acoustic behavior in $3 \mathrm{D}$ virtual environment [1][10] and potential benefits of acoustics simulation in construction industry.

\section{Hardware Configuration of the VE Walk-through System}

The virtual environment walk-through system is comprised of three major parts: visualization subsystem, auralization subsystem, and real-time interaction parts such as tracking systems that allow updating the position of the user's head and the virtual objects associated with virtual sound sources. The visualization subsystem (VE host) is centered on a CAVE that consists of a cube with display screen faces surrounding the user and driven by a SGI Onyx 2 rack system. The auralization subsystem is based on a Huron PCI audio workstation. A set of $\mathrm{TCP} / \mathrm{IP}$ protocol-based procedures was written to allow the VE host to transmit the attributes of the acoustic scene to the auralization subsystem through a local area network. The VE host sends packets specifying the acoustic scene, including the source, user and environmental attributes, achieved from the geometry of the building design (visual model). From these packets, the auralization host generates a pair of auralization filters and sends them to the DSP boards. The DSP board samples and processes an audio source with specified filters. Processed audio is then sent back to the VE area in analog form through two coaxial cables. The different parts of the system and their corresponding tasks are shown in Fig. 2.

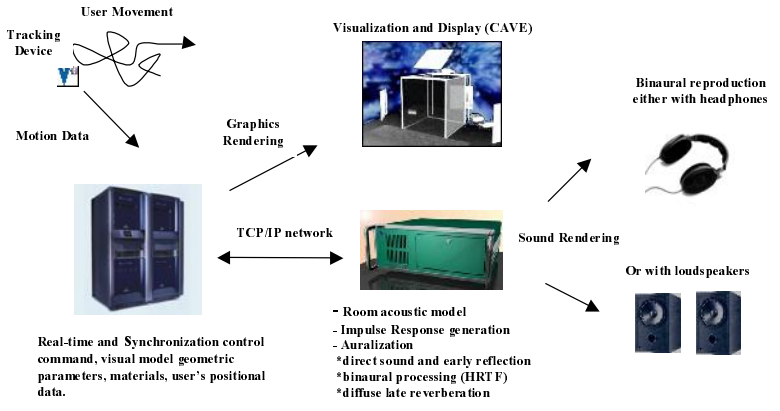

Fig. 2 Virtual Environment Walk-through System Architecture

\section{Software Architecture}

The software is a multi-thread parallel system. It works on SGI IRIX 6.5 system platform. It consists of Interface/Configuration Manager, World Manager, Input Manager, Viewer, Sound Manager and Database (see Fig. 3). The Interface/Configuration Manager gets the
CAD design, system configuration and parameters modification from a file or 3D widgets-GUI. It tracks all master processes to allow a run time configuration of the different modules.

The World Manager is responsible for administrating the overall system. It coordinates the visualization, user inputs, databases and sound management via network configured for TCP/IP protocol. The World Manager fetches the user input, makes format conversion considering the corresponding simulation constraints and simulation results, and passes the corresponding data (e.g. the position and orientation information of the user) to the Viewer and Sound Manager. These new data are used to update the scene graph and control the sound server via Sound Manager as well. The World Manager also has the responsibility to synchronize various threads.

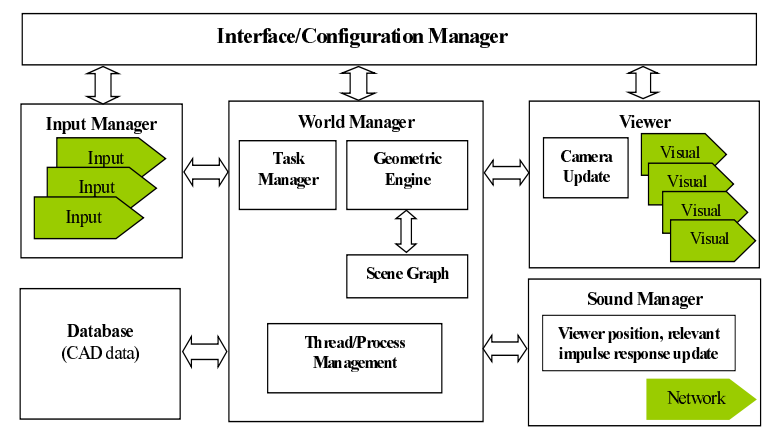

Fig. 3 Software Architecture

Extension to the OpenGL Optimizer toolkit has been made to view the scene in different display technology (e.g., CAVE, Reality Room, L-shape Workbench, desk top Monitor or other display). The Viewer renders the scene to the selected display faces in the appropriate mode. It is performed using parallel threads to provide real time response. The Configuration Manager passes this information to the Viewer, and according to the selected configuration the number of view cameras are created and the correct display type is initialized.

The Input Manager carries out user-object interactions, and it establishes the data flow between the user input and the object held by the World Manager. It supports devices such as tracking system, gloves and wands. These inputs describe the user actions/commands within the virtual environment. Each input modal has its own thread to process data from the input device. These threads run in parallel with the rendering threads to achieve low latency. Once the models are loaded into the system via CAD interface, the Input Manager allows the user to grab and manipulate objects in the 3D space.

The Sound Manager gets the traversed data of the listener (user/viewer) or sound sources and modified parameters from the World Manager and the user interface, and then uses the Huron API to manage the audio workstation via local network configured for 
TCP/IP protocol, for example, when the user moves around within the scene, it sends the relevant commands, which include the real time position and orientation information of the user, to the sound server. The sound server implements the real time sound rendering.

\section{Scenegraph Structure and Rendering}

The scene graph (virtual world) has been designed to maintain both geometric and polygonal data. Polygonal data is maintained to visualize the building models using standard polygonal rendering techniques. It has been created by the OpenGL Optimizer graphical toolkit. The scene graph structure is shown in Fig. 4.

The structure supports the direct interaction between the user and the components of the scene graph within the CAVE environment. There are significant differences between the interaction performed within a desktop environment, using 2D mouse-like devices, and direct interaction within immersive CAVE environments. In a desktop environment there is no spatial relation between the virtual world space and the real world space occupied by the user. However, in a CAVE environment, the physical CAVE space, in which the user is really acting, exists within the virtual world. The user may move this CAVE space within the virtual world space by using navigational device. In the virtual world representation of this system, the CAVE space is represented as the user space. The transformation node at the top of the user space allows the user to position the user space anywhere in the virtual world. In this way, the user is assumed to be always within the user space and his/her body position is tracked with respect to the center of the user space. The current user space structure maintains the head and the hand(s) positions within the user space. The tracker values are assigned directly to the hand and head transformation nodes to maintain their correct position within the virtual world.

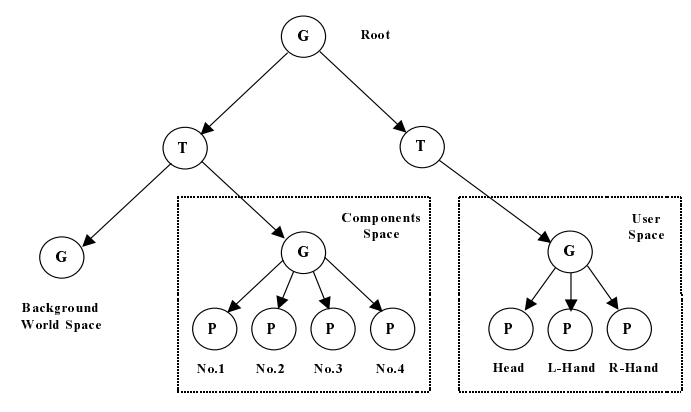

Fig. 4 Scene Graph Structure

The scene graph (virtual world) has two kinds of components, the background world space (buildings space) and moveable components space (furniture, electrical appliance space). The background world space is maintained to provide a visually realistic buildingwalk-through environment. The background world space is rendered but not available for interaction. The room acoustic models are associated with this space as well.
The moveable components can be associated with the corresponding virtual sound objects. So the sound sources and the visual models will moves together.

\section{Acoustic Environments Simulation and Rendering} The simulation of acoustic environments is a technique that has been used for a number of years in the field of acoustics. The ability to listen to a simulated acoustic space has enabled researcher in acoustics to examine the characteristics of a virtual or real acoustic space via computer modeling. Acoustic environment simulation is now being used as a reliable means of predicting the characteristics of an acoustic space that does not exist in reality. The method of simulation has become known as auralization [1], being the auditory counterpart to visualization.

Since the interaction of users with the virtual environment requires a sufficient real time behavior, computation time is limited. Due to the limitation of the computation time, the real time detailed auralization cannot be implemented, especially large and complex spaces. It is important to understand what aspects of the listening experience the user wish to gain for a given acoustic space, and then adopt the relevant trade-off algorithm to realize real time simplified acoustic space simulation or non-real time detailed acoustic simulation.

\subsection{Real-time Simplified Interactive Acoustic Simulation}

In order to implement real time virtual building walkthrough to experience the effect of the given acoustic space within the limited computation power, some trade-off algorithms have to be adopted. The Bformat soundfield representation is used since it is a convenient method for creating and manipulating 3D sound in auralization system. The B-format is essentially a four-channel audio format that can be recorded using a set of four coincident microphones arranged to provide one omni-directional channel (the $\mathrm{W}$ channel) and three figure-8 channels (the $\mathrm{X}, \mathrm{Y}, \mathrm{Z}$ channels). This set of $\mathrm{X}$, $\mathrm{Y}, \mathrm{Z}$ and $\mathrm{W}$ signals represent a first-order approximation to the soundfield at that point in space. Binaural impulse responses are used to simulate the room and headphone to display. The direct sound plus six first order reflections are calculated in real time and mixed to form the $\mathrm{X}, \mathrm{Y}, \mathrm{Z}, \mathrm{W}$ soundfield signals. The gain values used in this mixing are computed from the direction of arrival of each sound image at the user position. The volume of the geometry of the acoustic space is approximated by a box (shoebox) that is used to calculate the six first order reflection at run time. From the second order reflections to the reverberant tail of the room impulse response are pre-computed depend on the environmental parameters such as geometry of the room, materials of the room surfaces, locations/orientations of the sound sources/users etc. The binaural filters (based on Head Related Transfer Functions) do not change in real time. These filters are static. 


\subsection{Detailed Acoustic Simulation}

Non-real time detailed room impulse response is precomputed offline for pairs of points of sound source and user. It only supports user's orientation changing and walk-through along the pre-determined walking path within the virtual world in run time. It is used for critical evaluation of the room acoustic simulation. Two-stage filtering processes are used. The late part of the room impulse response is kept fixed and the early part of the room impulse response is selectively switched depending on the user's head orientation and position defined by a script-file.

\section{Unification of Visual and Audio Worlds}

The whole VE walk-through system consists of two parallel data streams: the visual stream and aural stream that the process from sound synthesis through sound propagation in a room to auralization in the user's ears. The two streams share some common control and synchronization mechanisms and information sources. The outputs of the streams are what the user will hear and see within the immersive virtual environment that this system creates. The visual aspects of the virtual environments emphasize in the geometry definition, motion description, and information stored about the physical properties of the world's structural materials are all oriented towards the requirements of visual rendering. The geometry and materials definitions evolved for visual virtual world are not always ideal for acoustic model that requires different geometric resolution and additional information about the structure of the world. The virtual world software was modified to extract the spatial coordinates of the user and sound source positions. This information is then coded as TCP/IP packets and transmitted to an audio server (aural stream) that runs a separate world model with the required acoustic properties. The audio server then spalialises the audio according to the geometry information received and introduces the appropriate room acoustics such as reflections and reverberation according to the room model loaded. It is capable of moving from room to room (walk-through) within the virtual world that allows the different room models to exhibit widely varying characteristics, such as a dead recording studio through to a large reverberant church.

The system components and the overall information flow are shown in Fig. 5. The upper half of the figure shows the audio stream while the lower half shows the visual stream. Visual models will be created using CAD tools, transformed and imported into this system using OpenGL Optimizer software.

\section{Cases Study}

For the real time simplified simulation, it is tested with a toy-room. Within the visual model, the toy room along with the bed, the wardrobe and the bookshelf belong to the background world space, which are not moveable. The television, telephone, radio, toy dog and toy helicopter are moveable components. They belong to moveable components space.

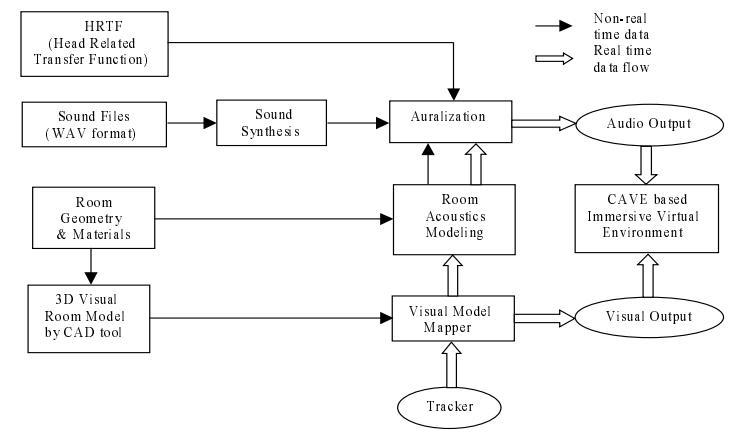

Fig. 5 The Virtual Environment Walk-through System Information Flowgraph

The sound objects are bound to them respectively. The user can walk around within the virtual room and turn the head at random; the system keeps the sound location static with respect to the physical environment, resulting in movement as the user turns their head.

It also supports interactivity. When the telephone is touched and picked up with virtual hand, it will ring. The sound object is bound to the telephone visual model. The user can move it far and near, up and down, left and right, back and forth, anywhere around himself or herself. The 3D sound bound to it will change in real time. The toy helicopter is flying in square track over the user's head and the user can hear the related 3D sound and smooth Doppler shift effect.

It also supports multiple sounds rendering. The user can interactively touch and move the television, radio, toy$\operatorname{dog}$ as well. When user moves them, the related 3D sound occurs in real time from their location within the virtual space. The sound streams are continuously updated as the user navigates around the virtual world, giving a perceptually compelling $3 \mathrm{D}$ soundfield. The photograph is shown in Fig. 6.

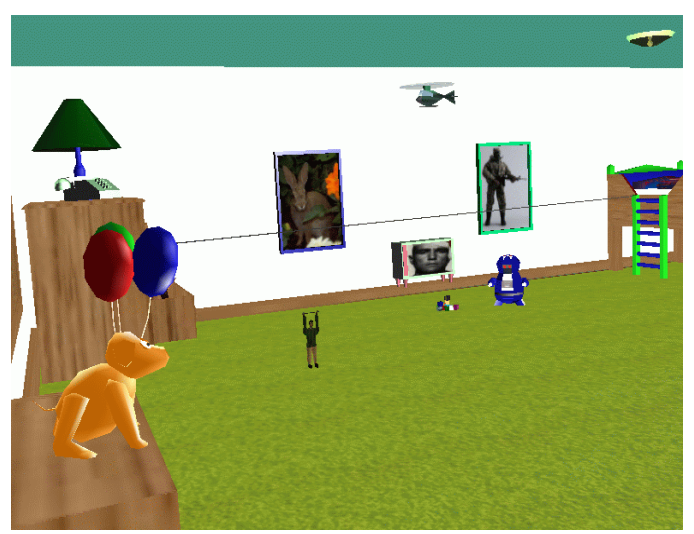

Fig. 6 The Toy Room Model

For the non-real time detailed simulation, it is tested with a cinema model. The detailed room impulse response pre-computed offline and then loaded in. It only supports 
real time walk around within the virtual space along a pre-determined walking path by a script-file and the user's head rotation via applying different HRTFs for different head positions. It can be used for critical evaluation of room acoustic simulation. The visual model of the cinema is shown in Fig. 7.

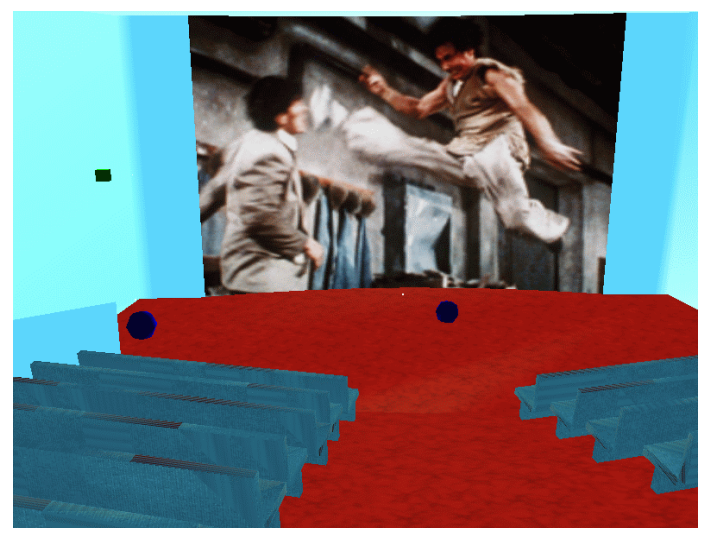

Fig. 7 The Cinema Model

\section{Conclusion}

A virtual environment walk-through system has been setup. The initial cases have been evaluated with the system and its trade-off algorithm for real time simplified acoustic space simulation via B-format soundfield representation, non-real time detailed acoustic space simulation and synchronous integration of auralization and visualization. Next step, the localization performance will be tested. In the future, we try to use the parallel method to achieve real time response and implement the parallel system instead of the distributed system that implements the sound rendering with an audio server. With the development of the componentbased software technology, we believe that the on-shelfcomponent of the auralization and visualization will be available, the flexibility and diversity will be improved significantly for implementation and integration of audiovisual virtual environment for construction applications.

\section{Acknowledgement}

Thanks give to Luis Marcelino, Norman Murray and Simon Campion in the Center for Virtual Environments for their contributions and suggestions.

\section{References}

[1] M. Kleiner, B.-I. Dalenback and P. Svensson, "Auralization--- An Overview", J. Audio Eng. Soc., Vol.41, No.11, 1993.
[2] J. Borish, "Extension of the Image Model to Arbitrary Polyhedra", J. Acoustic Soc. of America, Vol.75, No.6, 1984, pp. 1827-1836.

[3] R. Heinz, "Binaural Room Simulation Based on an Image Source Model with Diffuse Sound Scattering of Walls and to Prediction the Reverberant Tail", Applied Acoustics. Vol.38. 1993, pp. 148-159.

[4] T. Lewers, "A Combined Beam Tracing and Radiant Exchange Computer Model for Room Acoustics", Applied Acoustics, Vol.38, 1993, pp. 161-178.

[5] G.M. Naylor, "Odeon-Another Hybrid Room Acoustical Model", Applied Acoustics, Vol.38, 1993, pp. 131-143.

[6] T. Lokki, L. Savioja, R. Vaananen, J. Huopaniemi and T. Takala, "Creating Interactive Virtual Auditory Environments", IEEE Computer Graphics and Applications, Vol.22, No.4, July/August 2002.

[7] P. R. Cook, "Sound Production and Modeling", IEEE Computer Graphics and Applications, Vol.22, No.4, July/August 2002.

[8] Y. Zhang, T. Fernando and T.K. Wang, "Virtual Environment System for Interactive Acoustics Assess of Building", Proceeding of the ACM SIGGRAPH and EUROGRAPHICS Campfire: Acoustic Rendering for Virtual Environment, Snowbird, Utah, USA, May 2001.

[9] Y. Zhang, T. Fernando and L. Marcelino, "A Decision Support System for Interactive Acoustics Assess of Building Based on Virtual Environment", Proceeding of the $7^{\text {th }}$ CACSCUK'2001, Nottingham, England, 22 Sept. 2001, pp177-183. ISBN0953389049.

[10] T. Funkhouser, I. Carlbom, G. Elko, G. Pingali, M. Sondhi and J. West, "A Beam Tracing Approach to Acoustic Modeling for Interactive Virtual Environment", Proceeding of the ACM SIGGRAPH'98, pp. 21-23.

[11] K. Doel, P. G. Kry and D. K. Pai, "Physicallybased Sound Effects for Interactive Simulation and Animation", Proceeding of the ACM SIGGRAPH'2001, Los Angeles, CA, USA, August 2001.

[12] J. F. O'Brien, P.R. Cook and G. Essl, "Synthesizing Sounds from Physically Based Motion", Proceeding of the ACM SIGGRAPH'2001, Los Angeles, CA, USA, August 2001.

[13] E. Turban and J. E. Aronson, "Decision Support Systems and Intelligent Systems", $6^{\text {th }}$ ed. Prentice Hall International Inc., 2001.

[14] J. Vince, "Essential Virtual Reality fast", SpringerVerlag London Ltd., 1998. 